\title{
Modeling and Simulation of Quality of Service in VoIP Wireless LAN
}

\author{
A. Al-Naamany, H. Bourdoucen and W. Al-Menthari \\ Department of Electrical and Computer Engineering, Sultan Qaboos University, Al-Khodh, Muscat, Oman
}

This work presents computer modeling and simulation of the IEEE 802.11 standard to improve its Quality of Service (QoS) when different wireless stations are allowed for roaming. Request-to-Send and Clear-to-Send (RTS/CTS) frames are added as means to overcome the hidden node problem that could occur in wireless local area networks. The obtained simulation results are presented and discussed, including the ones for Quality of Service differentiation.

Keywords: Quality of Service (QoS), VoIP, WLAN, OPNET, IEEE 802.11e, roaming, RTS/CTS frames.

\section{Introduction}

Voice over Internet Protocol (VoIP) is a method used in data networks and broadband internet to establish voice calls. This is implemented by converting the analog voice calls into digital format that can be transmitted through the internet or through the intranet as shown in Figure 1.

As a result, voice signals will be transferred through a packet-switched network instead of

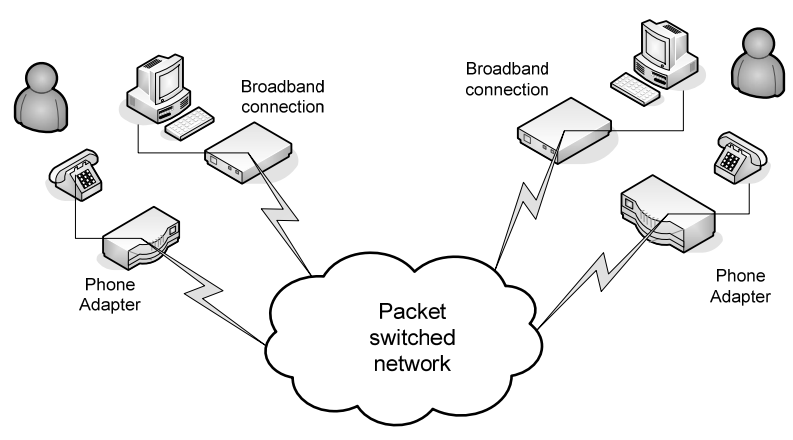

Figure 1. Diagram of VoIP transmission through the internet (adopted from reference [1]). being transmitted through a dedicated circuitswitched voice lines. This method gives an advantage of reducing and sometimes completely bypassing the expensive fees imposed by telephone companies. There are, for instance, many software packages that enable this type of calls through personal computers. Another method that performs this task is to use a telephone adapter which establishes this type of calls. [2]. It is worth noting that VoIP can be implemented in any IP-based network such as local area networks (LANs). With the introduction of WLAN, many manufacturers started testing the ability of using it for VoIP service [3]. Voice communication can thus benefit from the mobility offered by the network. After experimenting with this possibility, it was found that it is difficult to apply VoIP in a wireless environment. In such environments, the signal power at a certain point is difficult to determine, which leads to uncertainty in data rate. It is this pattern which creates difficulty in applying the Voice over IP in voice communication scheme.

Voice communication generally requires minimum specific time delay and a given dropped packet rate in order to have a good quality call. In order to implement VoIP in Wireless Local Area Networks (WLAN), it is required to utilize the available bandwidth in an optimal way to reach the desired quality level of transmission [4-5]. Hence, a good QoS is needed in WLAN to insure a reliable voice communication. It is thus a real challenge to implement a good QoS for Voice over WLAN, mainly due to bandwidth limitation. Previous work has been done on this subject concentrated on programming QoS in wireless stations within a BSS infrastructure [6]. In this paper, a similar subject is addressed 
with the addition of two features that were ignored previously. These are the stations' ability to roaming and addition of RTS/CTS management frames. Computer simulations performed will deal with the same assumptions as suggested in the previous work [6].

\section{Specifications of IEEE 802.11 Standards}

The 802.11 standard identifies the medium access control (MAC) and physical layer (PHY) for WLAN. There are two media access methods for 802.11 which are Distributed Coordinated Function (DCF) and Point Coordinated Function (PCF). Table 1 shows a comparison between these two methods [7].

\begin{tabular}{|l|l|}
\hline $\begin{array}{l}\text { Distributed Coordination } \\
\text { Function (DCF) }\end{array}$ & $\begin{array}{l}\text { Point Coordination } \\
\text { Function (PCF) }\end{array}$ \\
\hline \hline Best effort & Real time \\
\hline CSMA/CA & $\begin{array}{l}\text { Is connection oriented } \\
\text { (polling mechanism) }\end{array}$ \\
\hline $\begin{array}{l}\text { Uses contention } \\
\text { period (CP) }\end{array}$ & $\begin{array}{l}\text { Uses Contention Free } \\
\text { Period (CFP) }\end{array}$ \\
\hline $\begin{array}{l}\text { - Assures Soft QoS in } \\
\text { - May fail to allocate } \\
\text { channel resources to } \\
\text { competing stations } \\
\text { - No grantee in BW, } \\
\text { packet delay and jitter } \\
\text { - Throughput degrada- } \\
\text { tion in heavy load }\end{array}$ & $\begin{array}{l}\text { - Works nicely in a } \\
\text { network with one AP only } \\
\text { as a result of using } \\
\text { multiple AP } \\
\text { - Overlapping cell problem } \\
\text { (when having more AP) } \\
\text { - Unknown transmission } \\
\text { time of polled station }\end{array}$ \\
\hline
\end{tabular}

Table 1. Comparison between DCF \& PCF (adopted from [8]).

One of the main tasks of the $802.11 \mathrm{e}$ is to investigate the possibility of implementing QoS in WLAN in order to create an environment suitable for applying high demanding applications like voice and video. A new coordination function is presented in 802.11e. It is called the Hybrid Coordination Function (HCF) and it uses the DCF as building block for its operation. It consists of three access methods, Enhanced Distributed Channel Access (EDCA), HCF Controlled Channel Access (HCCA) and PCF. Each method tackles the traffic differently, in order to provide the QoS needed. EDCA gives high priority to some traffic and low priority to others (prioritized QoS). This way, higher priority packets succeed in accessing the wireless medium more frequently than lower priority packets. The main problem is that it does not guarantee a certain bandwidth (BW) or protects it from diminishing due to a node addition or a heavy load. On the other hand, HCCA reserves the bandwidth by assessing and controlling which holds the BW for certain time i.e. parameterized QoS. The HCCA stream requirements are scheduled and held, therefore they are never interrupted regardless of what other types of traffic or devices are introduced to the network. Enhanced Distributed Channel Access is used in CP for channel access while HCCA is mostly used in the CFP [9].

\section{Aim of Work and Assumptions Used}

The aim of this work is to program and simulate an Enhanced Distributed Channel Access (EDCA) data priority technique of the 802.11e standard, using the existing OPNET WLAN station. However, in order to implement the EDCA design within the existing WLAN station, different OPNET editors need to be used to achieve the desired QoS architecture [10]. As a result, the MAC layer of the newly redesigned WLAN station will have the ability to organize the data arrived from the higher layer and assign the priority to each traffic type according to the preset requirements. This priority will affect the time required to gain access to the wireless medium. The aim is to create four levels of priority as shown below:

1. Interactive voice having the first priority.

2. Streaming multimedia with a second priority.

3. Background of third priority.

4. Best efforts of fourth priority.

Thus, wireless network stations will be allowed to roam and access the different points for handover. In addition, the hidden node problems will be considered by enabling RTS/CTS management frames.

\subsection{Assumptions Used}

Throughout the simulation, the considered wireless network was kept within the infrastructure and not with the Ad-hoc mode using access 
points (APs). The connection to the wired LAN was ignored since the AP took care of all the packets forwarded to the wired LAN. Table 2 presents some standard parameters that are common to different wireless stations used in the simulation.

\begin{tabular}{|c|c|c|}
\hline Parameter & Value & Description \\
\hline $\begin{array}{c}\text { Physical } \\
\text { characteristic }\end{array}$ & $\begin{array}{l}\text { Direct } \\
\text { sequence }\end{array}$ & $802.11 b$ \\
\hline Data rate & $5.5 \mathrm{Mbps}$ & $\backslash$ \\
\hline Transmit power & 0.005 Watts & $\backslash$ \\
\hline Slot time & $20 \mu \mathrm{sec}$ & $\begin{array}{l}\text { Standard } \\
\text { measurement } \\
\text { for other } \\
\text { parameters } \\
\text { like backoff }\end{array}$ \\
\hline SIFS & $10 \mu \mathrm{sec}$ & $\begin{array}{l}\text { Shortest IFS } \\
\text { and used for } \\
\text { ACK and CTS }\end{array}$ \\
\hline PCF parameters & Enabled & $\longrightarrow$ \\
\hline $\begin{array}{l}\text { Destination } \\
\text { address }\end{array}$ & Random & $\begin{array}{l}\text { Packets will } \\
\text { be transmitted } \\
\text { randomly to } \\
\text { different users }\end{array}$ \\
\hline $\begin{array}{l}\text { Roaming } \\
\text { capability }\end{array}$ & Disabled & $\backslash$ \\
\hline $\begin{array}{l}\text { Packet reception } \\
\text { power threshold }\end{array}$ & $-95 \mathrm{dBm}$ & $\begin{array}{l}\text { Receiver } \\
\text { sensitivity }\end{array}$ \\
\hline Source type & Bursty source & 1 \\
\hline Packet size & 1024 bytes & $\begin{array}{l}\text { Size of packets } \\
\text { generated by } \\
\text { the node source }\end{array}$ \\
\hline $\begin{array}{l}\text { Inter arrival } \\
\text { time }\end{array}$ & $0.020 \mathrm{sec}$ & $\begin{array}{l}\text { Time between } \\
\text { two successive } \\
\text { packet generated } \\
\text { by the station }\end{array}$ \\
\hline
\end{tabular}

Table 2. Typical simulation parameters used.

It is worth noting that many statistics can be collected after simulating any design in OPNET: media access delay, throughput, load, number of backoff slots and number of retransmission attempts.

Media access delay is the total number of queue and contention delays of data packets received by WLAN MAC from higher layer. The load statistic for the AP is an indication for the number of data traffic successfully received and forwarded to the destination in the wireless network. The AP load is an indication of its utilization by other stations. In this paper only the media access delay and the load will be considered and presented.

\section{Simulation Results}

Initially, four types of data are simulated without implementing the QoS design in the WLAN station. Then, these four data types will be simulated again after implementing the programmed QoS. After that, different simulation phases are carried out to test the performance of the newly programmed QoS.

For each of the above scenarios, the simulation will proceed with different parameters as indicated below:

- No stations' roaming and no RTS/CTS frames enabled.

- Stations' roaming is enabled and RTS has the threshold of 256 bytes.

- Roaming will be retained and RTS threshold increased to 1024 bytes.

The intention is to test WLAN performance with the existence of real-life factors, such as roaming and hidden node problems.

Note that OPNET Modeler version 11.0 was used to program and simulate these different scenarios. The simulation results that were collected, and evaluated will be presented and discussed in the following sections.

\subsection{Scenario 1: Simulation of WLAN without 802.11e Protocol}

The objective of this part is to simulate a normal wireless network without any quality of service. Four types of services were introduced in the network for the purpose of measuring their performance in normal conditions, as shown in Figure 2.

The minimum contention window value used was 31 backoff slots, while the maximum equals 1023 slots, where each station sends data randomly to different hosts. Note that the rate of the packet generation within the station was for all four types of services. The reason behind this is to create an environment where all the stations generate packets for the four types of services at the same rate. The rate of packets 


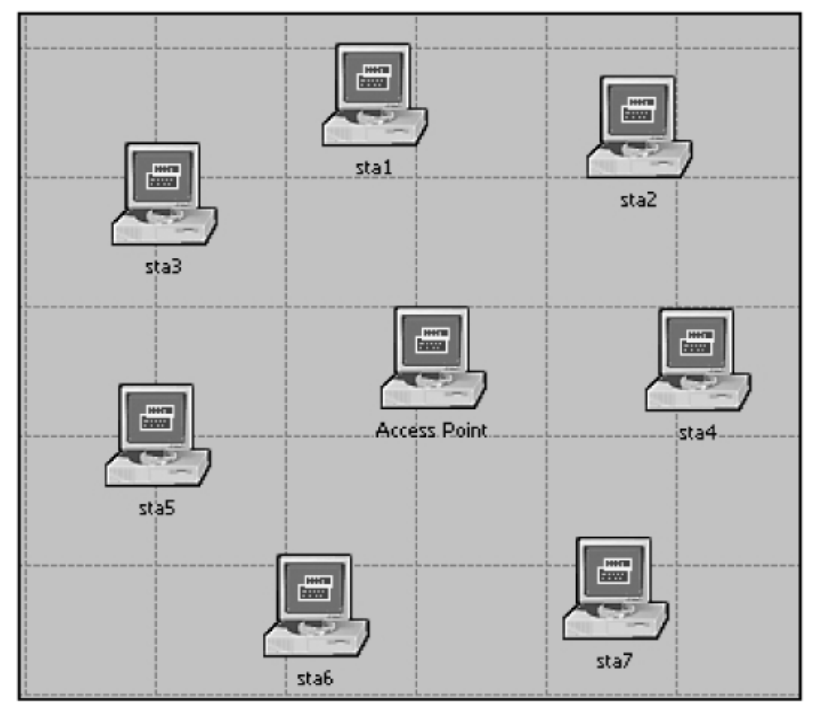

Figure 2. WLAN without QoS.

transmission, on the other hand, was kept random and depended only on the availability of the channel. The simulation period was calculated to be 120 seconds in OPNET time. By taking the average of the results collected for the media access delay, the number of backoff slots and the throughput, 120 seconds were enough to give an idea about the performance for each type of service.

For the first case, when stations' roaming is disabled and no RTS frames are added, the media access delay statistics show that all the four traffic types have the same rate at around 0.81 msec, as illustrated in Figure 3. This result was expected as there is no means of favoring one service type over another. All the four types of services were treated equally and had the same delay before they were allowed to send data.

In the second case, roaming was enabled and RTS threshold was set to 256 bytes. The statistics obtained show that the rate was equal for all four types of traffic. However, the performance was degraded as the media access delay reached $1.1 \mathrm{msec}$, as shown in Figure 4. This was expected, since roaming of the stations would result in an increased collision rate and therefore generate more competition on the available media, which causes a longer delay. Moreover, the added RTS consumes an important amount of capacity and overhead, resulting in additional latency. The value of RTS threshold causes RTS packets to be sent more often, which consumes more bandwidth and therefore reduces the throughput of other network packets.
The network was also simulated with RTS threshold of 1024 bytes. The performance improved slightly with respect to that of the simulation with no roaming and no RTS/CTS with a resulting media access delay of $0.80 \mathrm{msec}$. This improvement was due to the large value of RTS threshold, where the fewer retransmissions reduced the overhead.

This value allows one node to send its message almost entirely without interruption and without

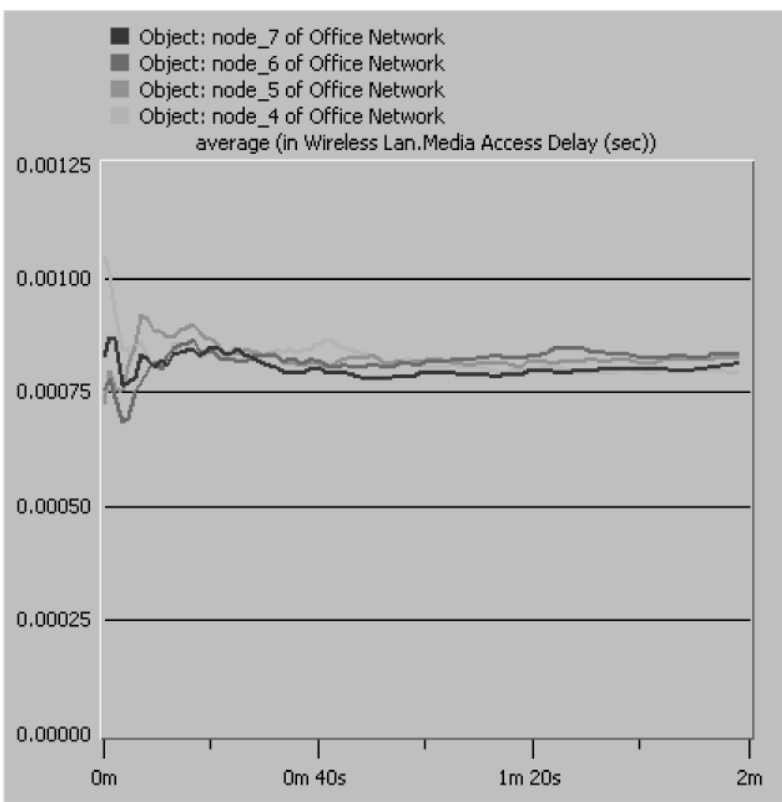

Figure 3. Media access delay without QoS, where roaming and RTS are disabled.

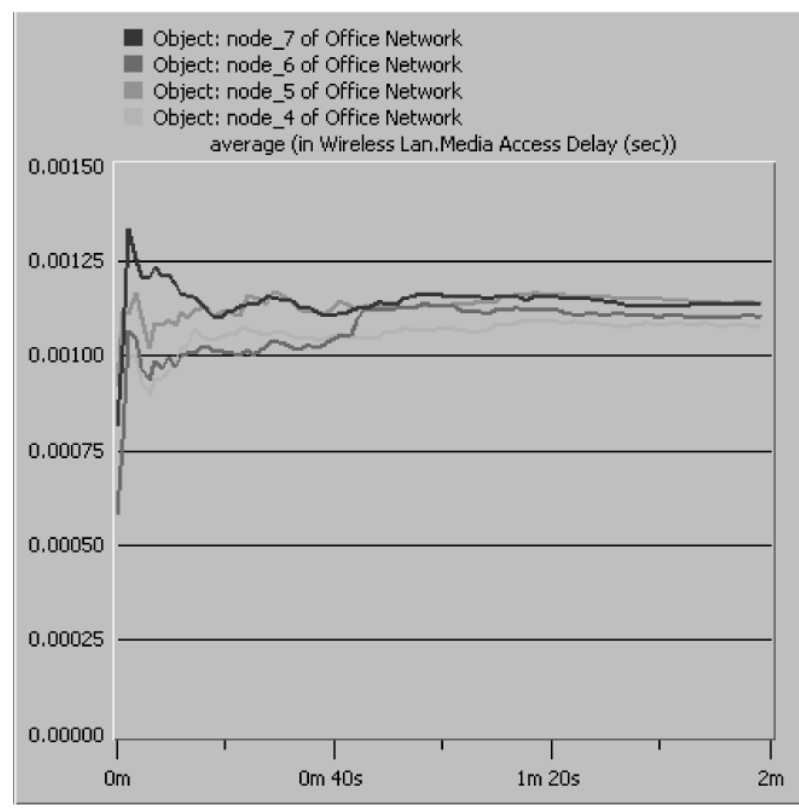

Figure 4. Media access delay without QoS, with enabled roaming and RTS threshold of 256 bytes. 


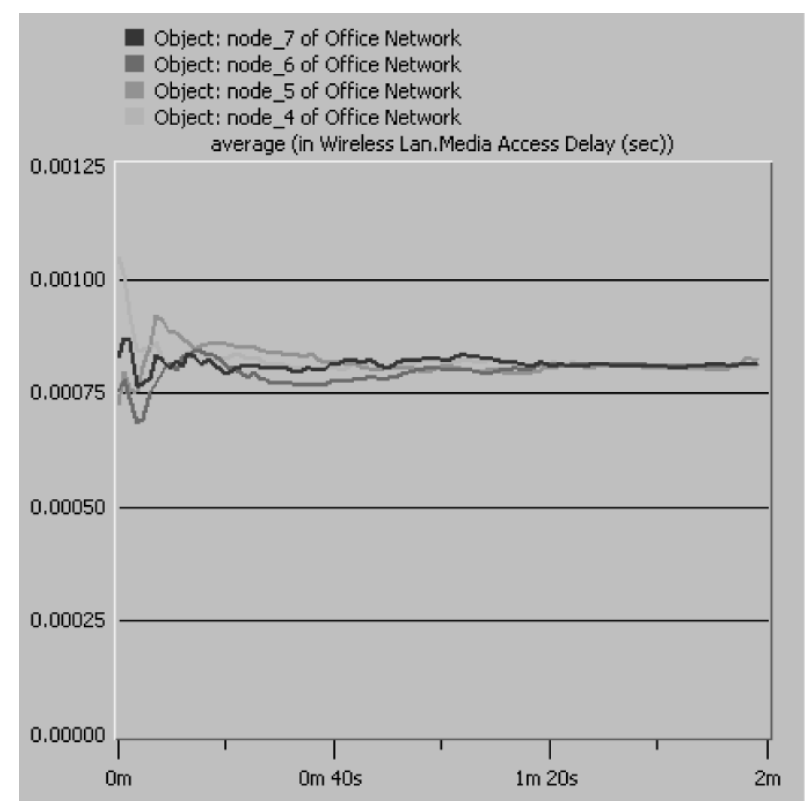

Figure 5. Media access delay without QoS, with enabled roaming and RTS threshold of 1024 bytes.

a need to stop and restart again. i.e. without consuming too much unnecessary bandwidth. This is why one needs to focus on the case where no roaming and no RTS/CTS threshold are available in the scenarios. The other case to be considered is when roaming is enabled and the RTS has a threshold of 1024 bytes.

\subsection{Scenario \#2: Using 802.11e Protocol}

The layout of the wireless nodes (stations) from the previous scenario was maintained. The difference was that the 802.11 e protocol was programmed and implemented in the second layer. The results were collected for each data type. The simulation durations were ranging from one to two minutes in OPNET time. It was set that each data type would be generated at the same rate and with the same generation parameters. The purpose of this setup was to compare the performance of the four data types when accessing the media, rather than their generation speed.

The media access delay results performed as expected according to the contentions window and AIFS parameters set. The voice traffic performed better than the others with the minimum media access delay at around $0.150 \mathrm{msec}$. The lower media access delay value, the faster the traffic access to the wireless medium. This also results in a higher priority traffic compared to the others. The second priority goes to the multimedia with the media access delay at 0.168 msec. After these two comes the background and the best effort traffic at 0.257 and 0.344 msec respectively. These delays are shown in Figure 6.

As for the case where the roaming was enabled for different stations and RTS threshold was set to 1024 bytes, it was found that media access

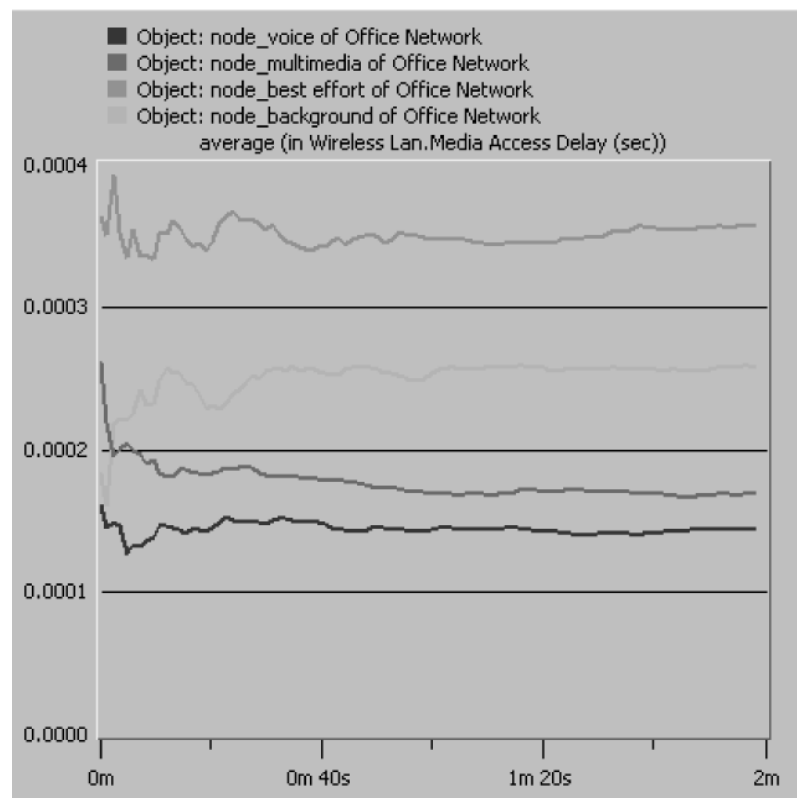

Figure 6. Media access delay of the four traffic types with QoS.

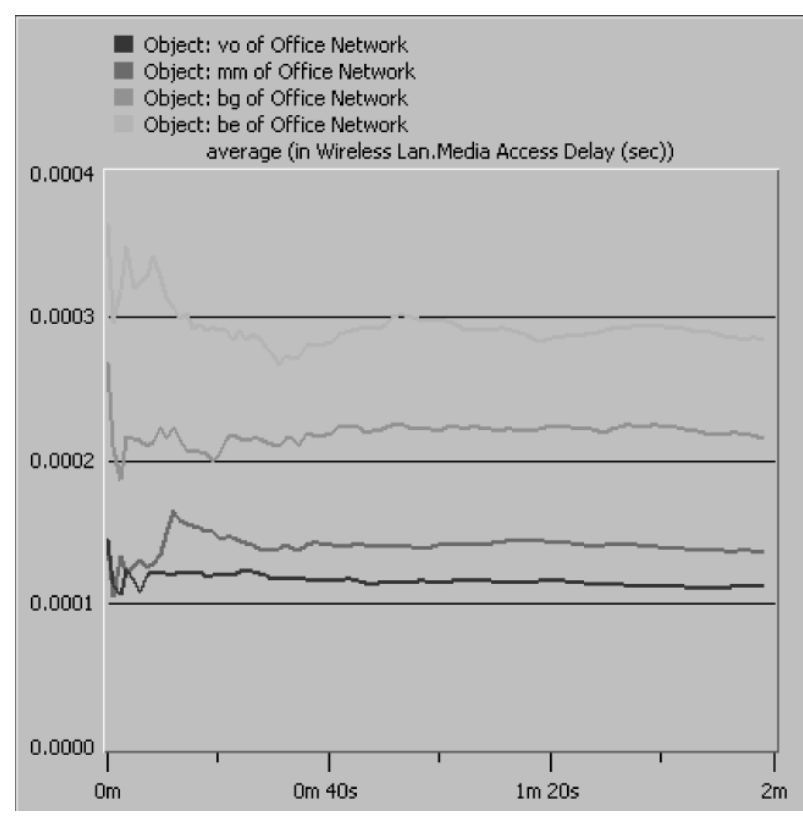

Figure 7. Media access delay of the four traffic types with QoS with enabled roaming and RTS threshold of 1024 bytes. 
delay for voice traffic was $0.111 \mathrm{msec}$. This indicates that voice traffic performance is improved with the introduction of RTS/CTS control frames of 1024 bytes. The improvement is again due to the reasons mentioned above. The obtained simulation results are shown in Figure 7.

Having compared the four traffic types in both cases, the load on the AP was examined. AP load indicated the instantaneous number of packets served by the AP. It was found to be around 178 packets when both roaming and RTS were disabled, compared to 175 when both of them were enabled. This result indicates that the AP utilization in the second case is slightly

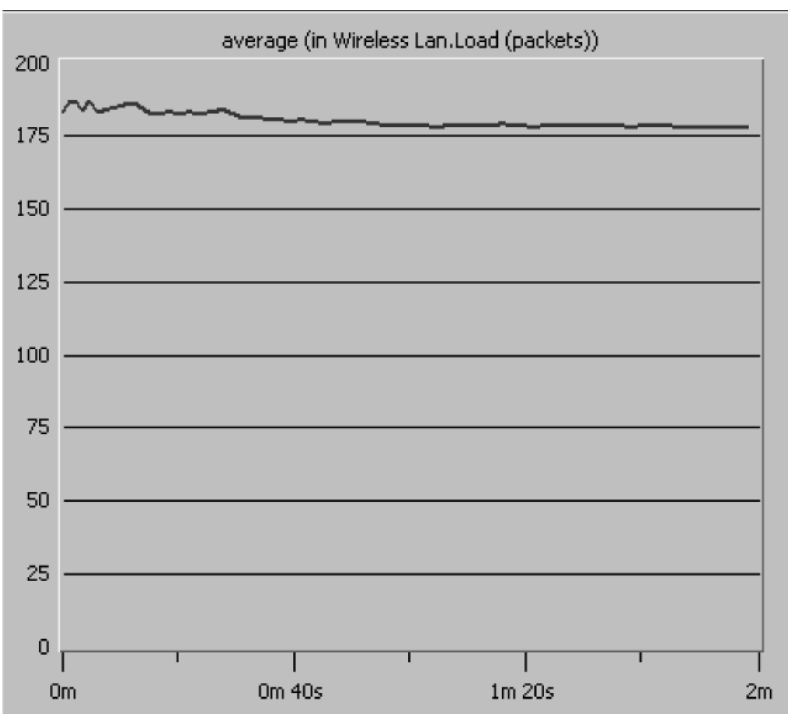

Figure 8. Load on the access point where roaming and RTS are disabled.

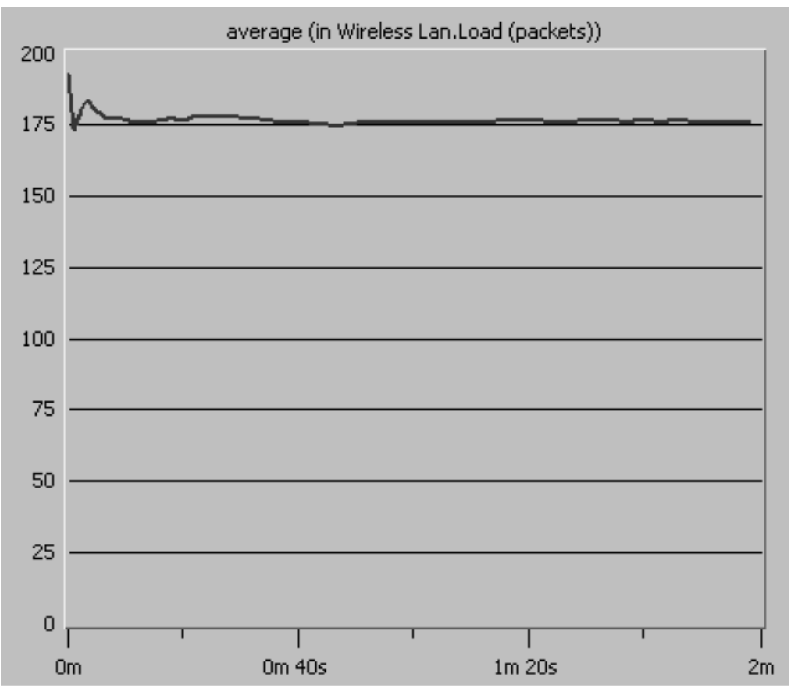

Figure 9. Load on the access point with enabled roaming and RTS. lighter than it is in the first case. However, these two results are close to each other, which indicates that enabling roaming and RTS in WLAN solely affects the stations, but not the AP. The AP loads for both cases are shown in Figures 8 and 9 respectively.

\subsection{Scenario \#3: Simulation of Heavy Load Wireless Network}

The heavy load wireless network was arranged by increasing the number of stations within the wireless network. The setup resulted in an increased number of attempts to access the wireless medium. The objective of this simulation was to test and examine the four traffic types in case of bottlenecks. The purpose of the simulation was to check if the traffic types still maintained the same level of priority in this situation. Its duration was one minute in OPNET time. It was set in such a way that each data type was generated at the same rate and with the same generation parameters.

The overall media access delay for the four traffic types increased under heavy load. This result was expected since the chance of getting a free medium was less this time. The traffic performed well, even in this situation of maintaining the same priority schemes. The voice was the lowest at $0.305 \mathrm{msec}$ and the best effort

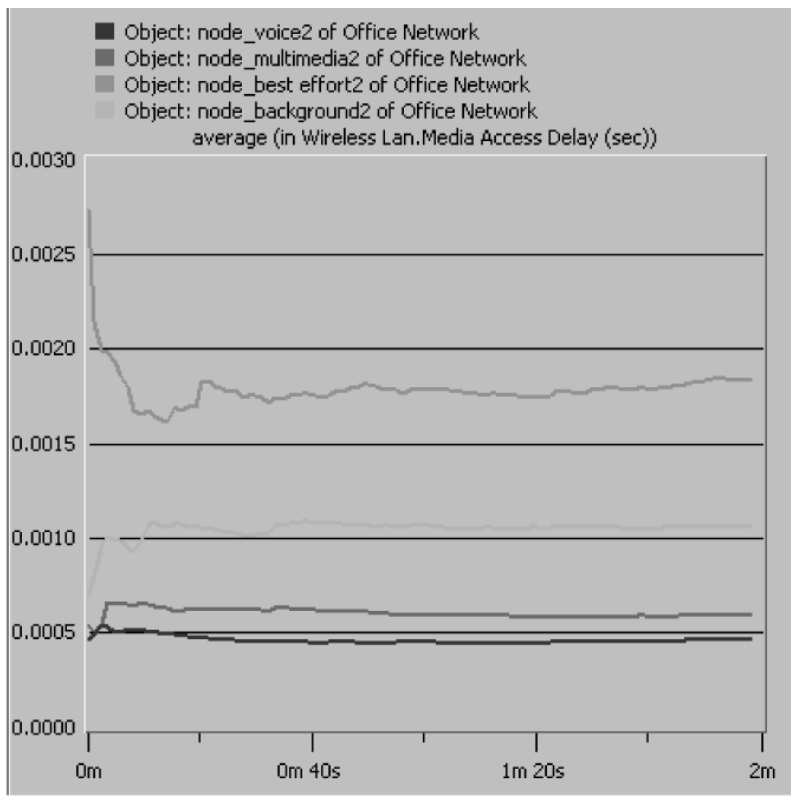

Figure 10. Media access delay under heavy load, disabled roaming and RTS. 
was the highest at around $01.284 \mathrm{msec}$ (Figure 10).

Figure 11 shows the simulation results with enabled roaming and RTS threshold of 1024 bytes in heavy load environment. The priority is maintained as the voice has the lowest delay of $0.313 \mathrm{msec}$ followed by multimedia and then background and finally best effort with a delay of $1.308 \mathrm{msec}$.

However, comparing these results with those obtained when neither roaming nor RTS were utilized, one can notice that multimedia delay is less in the second case than in the first one, while with all remaining types, delays were higher in the second case. The reason is that, under heavy load, more stations would saturate the available bandwidth, which results in more collisions.

Applying roaming stations and large threshold of RTS frames slightly affects the network performance.

The load on the AP, shown in Figure 12, increased from 178 packets to nearly 414 in the first case. However, in the second case, shown in Figure 13, the AP load also increased from 177 to 415 packets. The results obtained regarding the AP load in heavily loaded cases are comparable. This net increase appears to be the result of the increase in the traffic sent.

The network maintains good priority features under heavy load and the overall results were

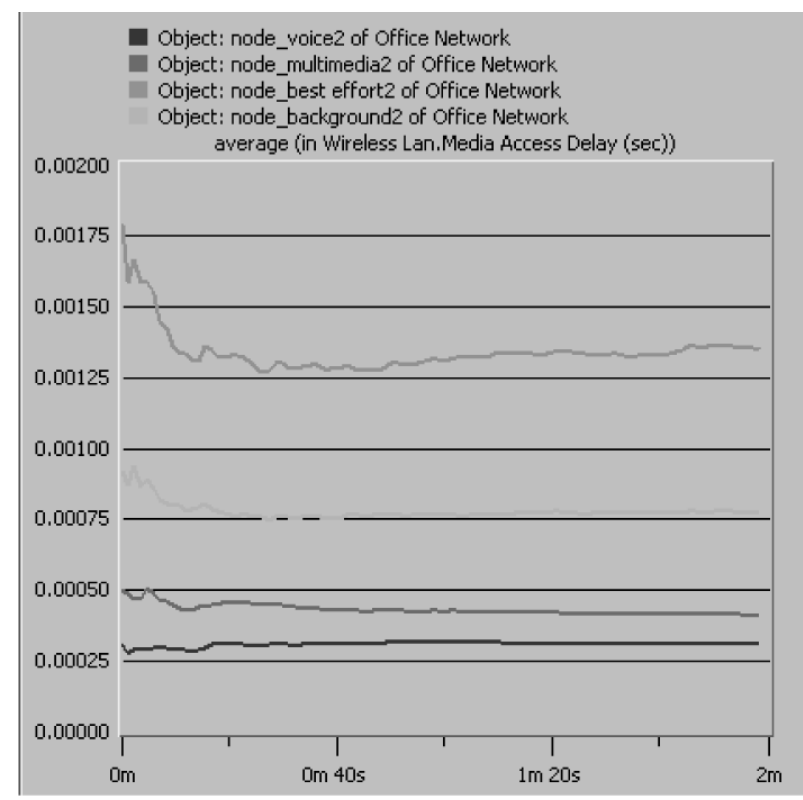

Figure 11. Media access delay under heavy load, with enabled roaming and RTS. affected noticeably because of the high traffic rate. The voice traffic performed well, as expected, and the performance of the multimedia traffic was below the voice, but still higher than the best effort and background.

\subsection{Scenario \#4: Simulation of Heavy Load Environment Using Multiple Access Points (APs)}

The objective of this simulation was to experiment with the results collected from the previous simulation to show that by carefully designing a WLAN with enough bandwidth and coverage, one can overcome some of the problems

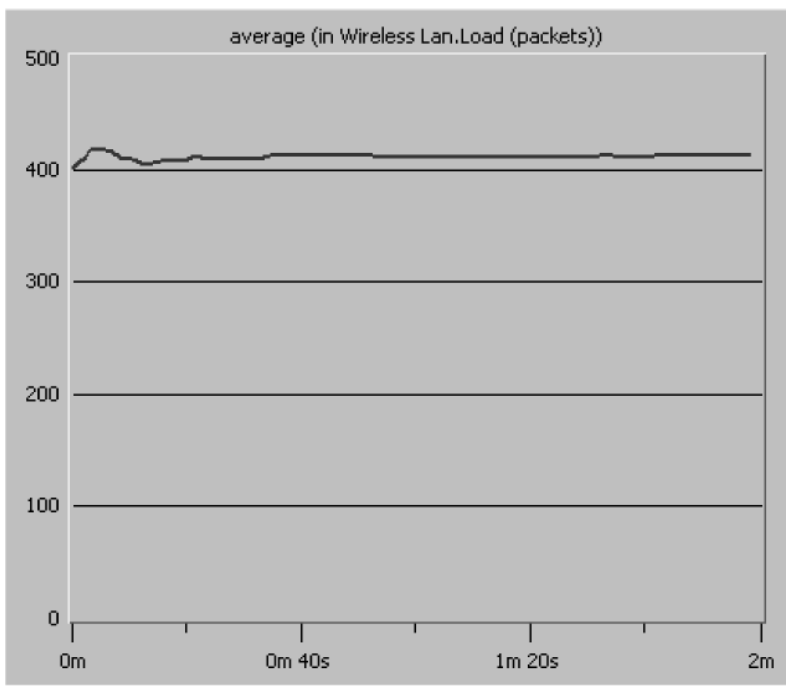

Figure 12. Load of Access Point in heavy load, with disabled roaming and RTS.

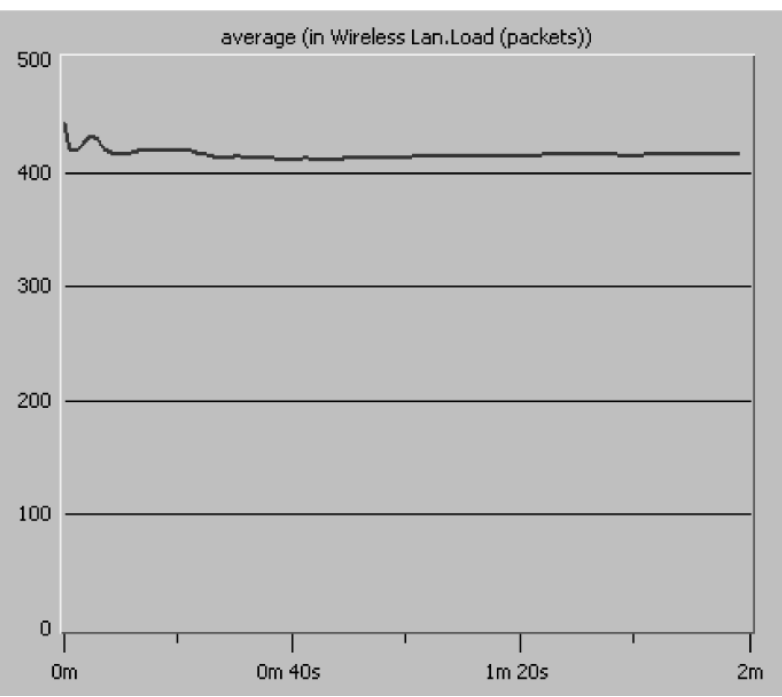

Figure 13. Load of AP in heavy load, with enabled roaming and RTS. 
that the network might be facing. In this simulation, two APs were introduced in the same increased load wireless network. This network was simulated for about one minute in OPNET time and was arranged in such a way that the stations was distributed evenly between the two APs.

It has been noticed from the media access delay results shown in Figure 14 that the levels are much closer to the levels of media access delay before applying the heavy load. The priority was not affected by introducing a new AP and the traffic levels were between $0.212 \mathrm{msec}$ and $0.083 \mathrm{msec}$ whereas the voice packets were still maintaining the highest priority.

Applying stations roaming and allowing RTS/ CTS frames with threshold of 1024 results in almost the same outcomes as those obtained before applying these changes, where voice traffic has delay of $0.083 \mathrm{msec}$ and best effort traffic has $0.201 \mathrm{msec}$ delay (refer to Figure 15).

The similarity is caused by the presence of the two APs. This means that the stations will be roaming from one BBS to another, and each BBS will be affected by almost the same factors.

By introducing another AP into the network, the load will be distributed evenly between the two APs. Each wireless station will then connect to the AP with the highest signal power. It is worth noting that the closer the station to

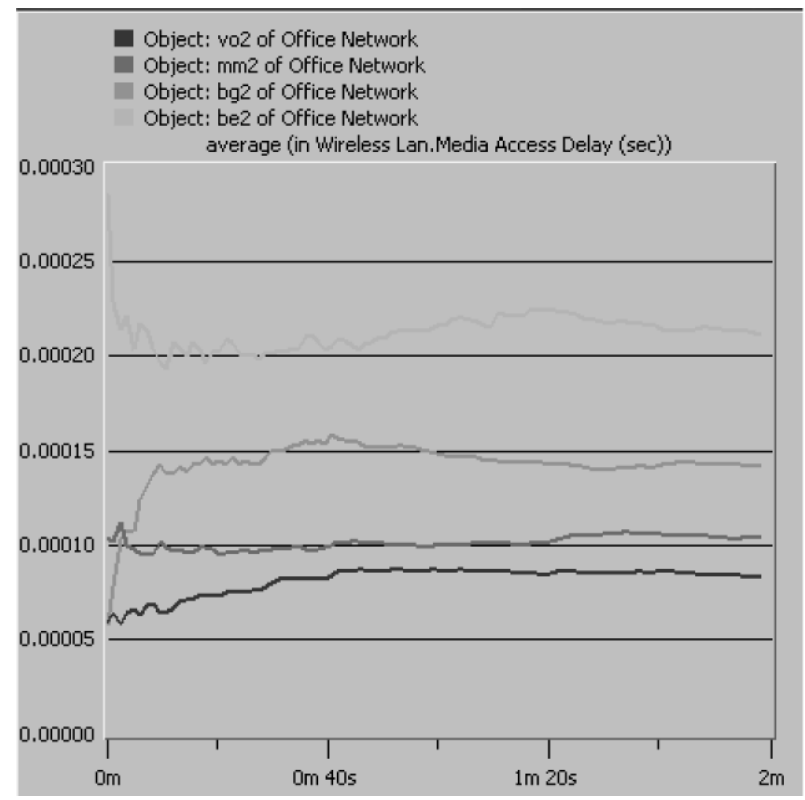

Figure 14. Media access delay with two AP with disabled roaming and RTS. get to the AP, the higher the power of the signal transmitted. As a result, most stations will connect to the closer AP. Figure 16 shows the distribution of the loads between the two APs.

Figure 17 shows the load which is distributed between the two access points and appears to be almost equal. One AP has a load of 80 packets whereas the other has a load of 77 packets. It can be noticed that introduction of RTS does not affect the load performance significantly.

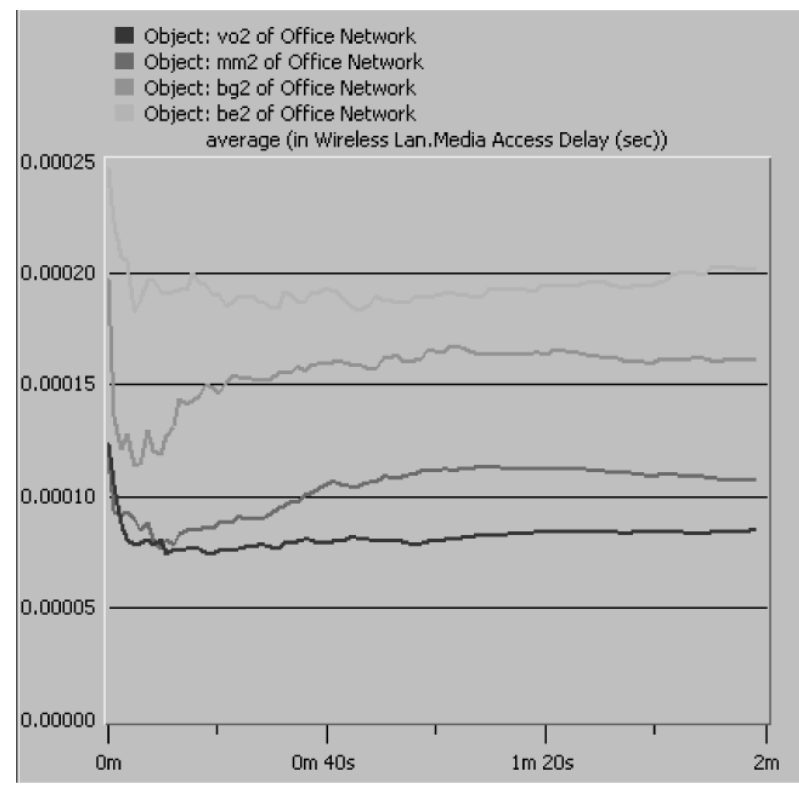

Figure 15. Media access delay with 2 AP with enabled roaming and RTS.

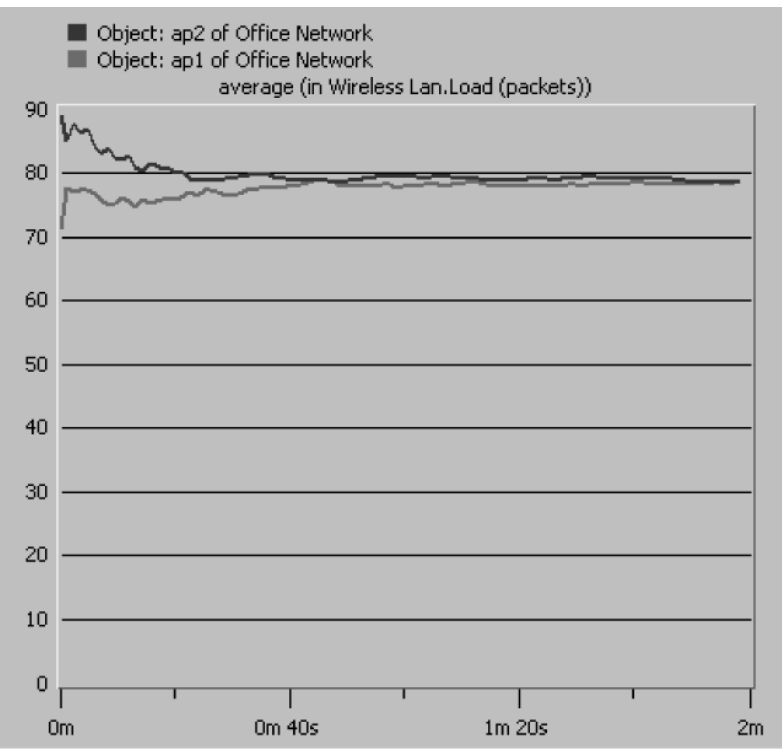

Figure 16. Load of the two AP with disabled roaming and RTS. 


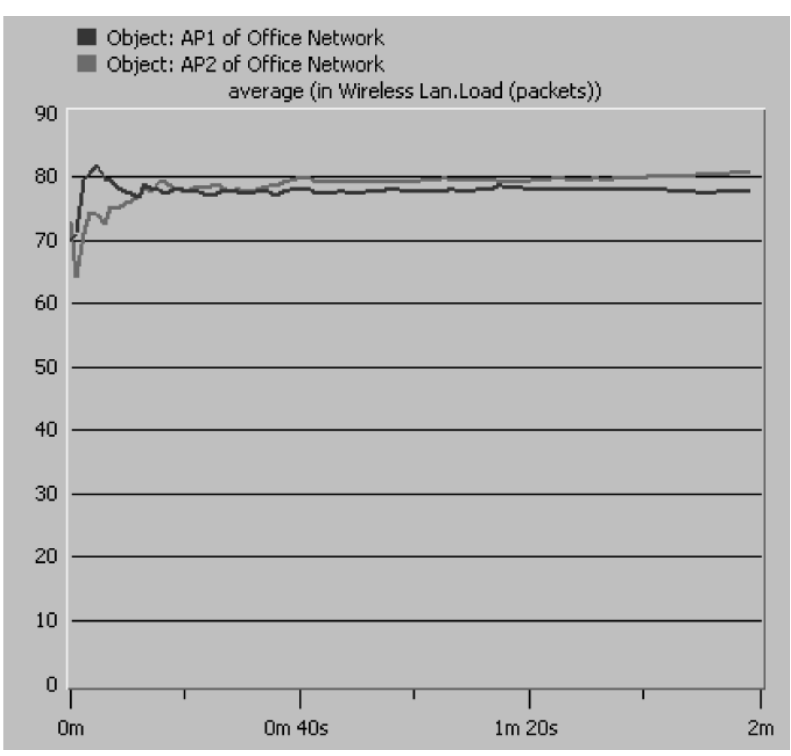

Figure 17. Load of the two AP with enabled roaming and RTS.

The introduction of a new AP to the network stabilized most of the extreme changes occuring as a result of the heavy load. The new $\mathrm{AP}$ acts as a new channel for data stream to be moved from the source to the destination. It is therefore very important to carefully design a wireless network with enough coverage and enough bandwidth to reduce the possibility of congestions and collisions.

\subsection{Scenario \#5: Voice over Wireless Area Network (VoWLAN)}

This is the final simulation which basically compares between two wireless networks with voice over IP handsets as illustrated in Figure 18. Note that both networks were simulated two times. The first time, neither roaming nor RTS were used and the second time, both were applied to each of the two networks.

The difference between these two networks is the implementation of quality of service. The first network is without any QoS, while the other has a QoS implemented. Performance of the voice handsets is compared for the two networks in terms of Media Access Delay. In order to simulate a voice handset in the network, a modified station was used to function as a voice handset. These modifications were written in the source that generated the packets within the station's node model. The inter-arrival time between the packets was also changed to transmit at a rate close to the real voice handsets rate. Moreover, the voice handset communicated only with other voice handsets that were implemented by setting the destination address to similar voice handsets. Other stations in the network were supposed to transmit randomly to all other stations. Note that the network was arranged to have seven stations and three voice headsets.

When no roaming and no RS were applied, VoIP sets performed as expected. The media access delay values were performing better

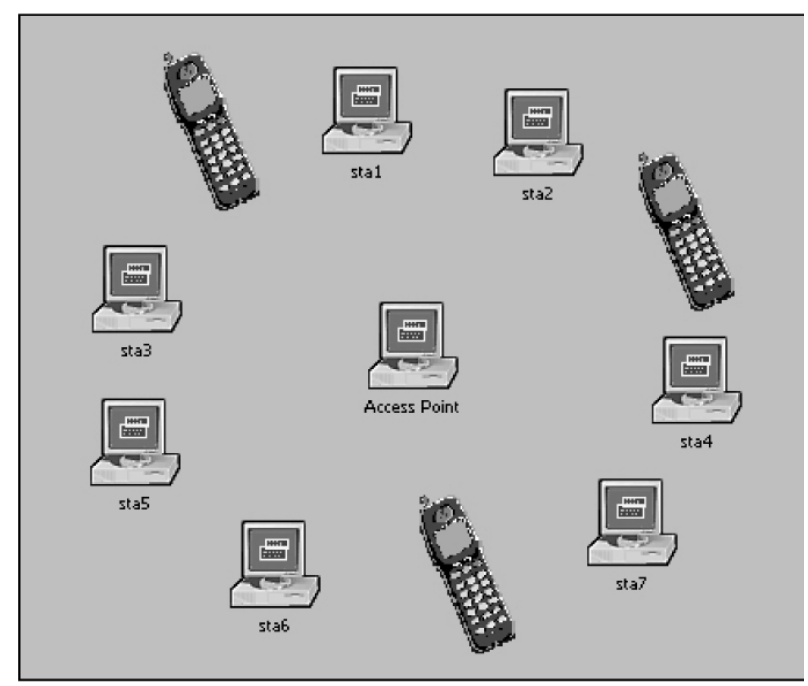

Figure 18. Voice over Wireless Local Area Network.

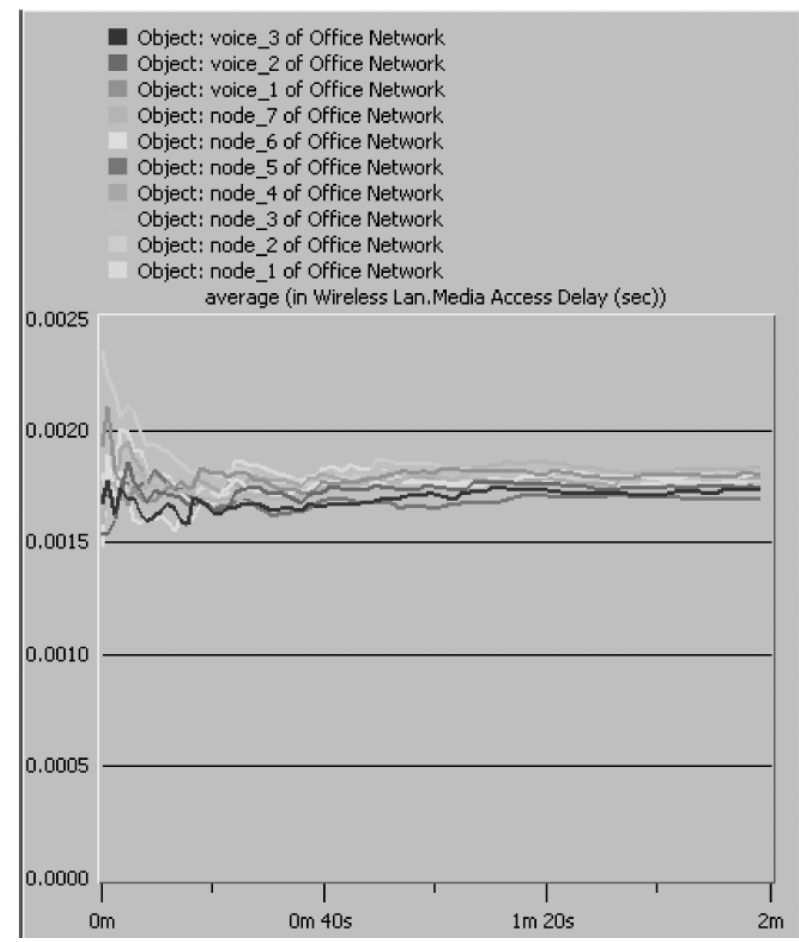

Figure 19. Media access delay without QoS. 
when the QoS was introduced in the second network. These results are illustrated in Figures 19 and 20.

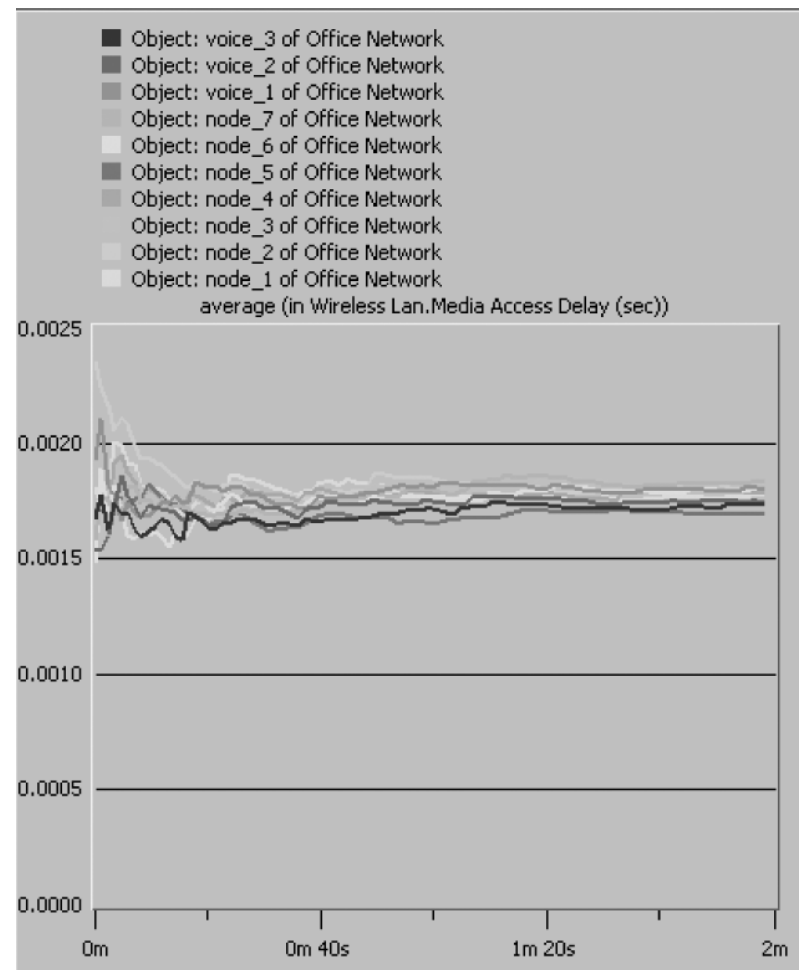

Figure 20. Media access delay without QoS, with roaming and RTS.

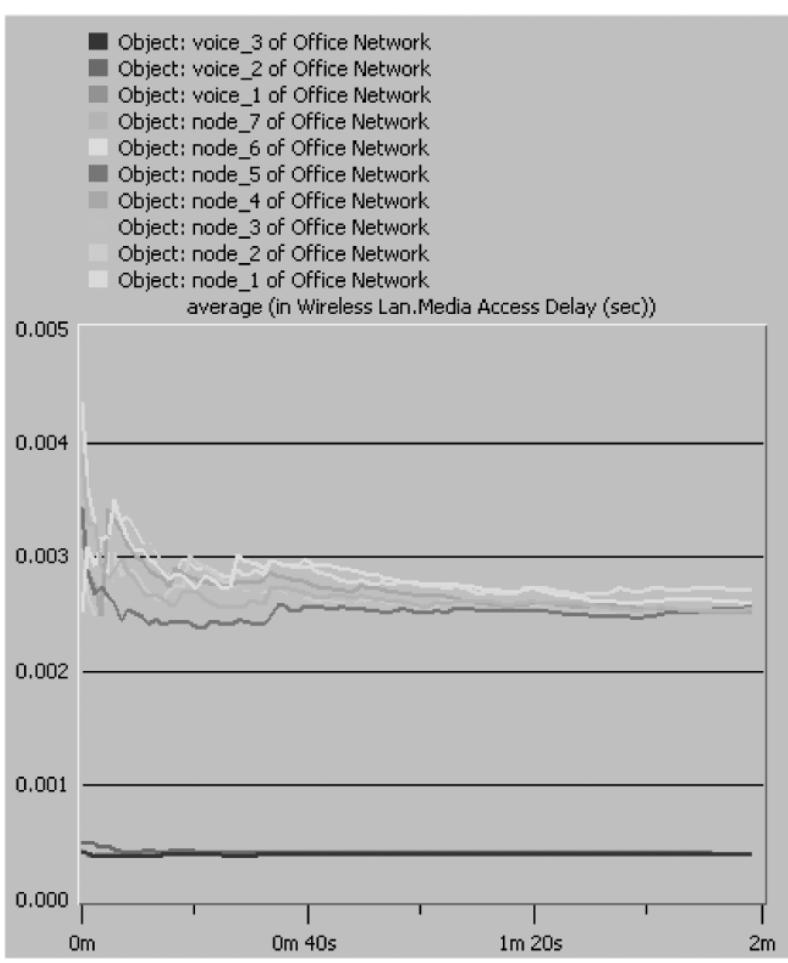

Figure 21. Media access delay with QoS.

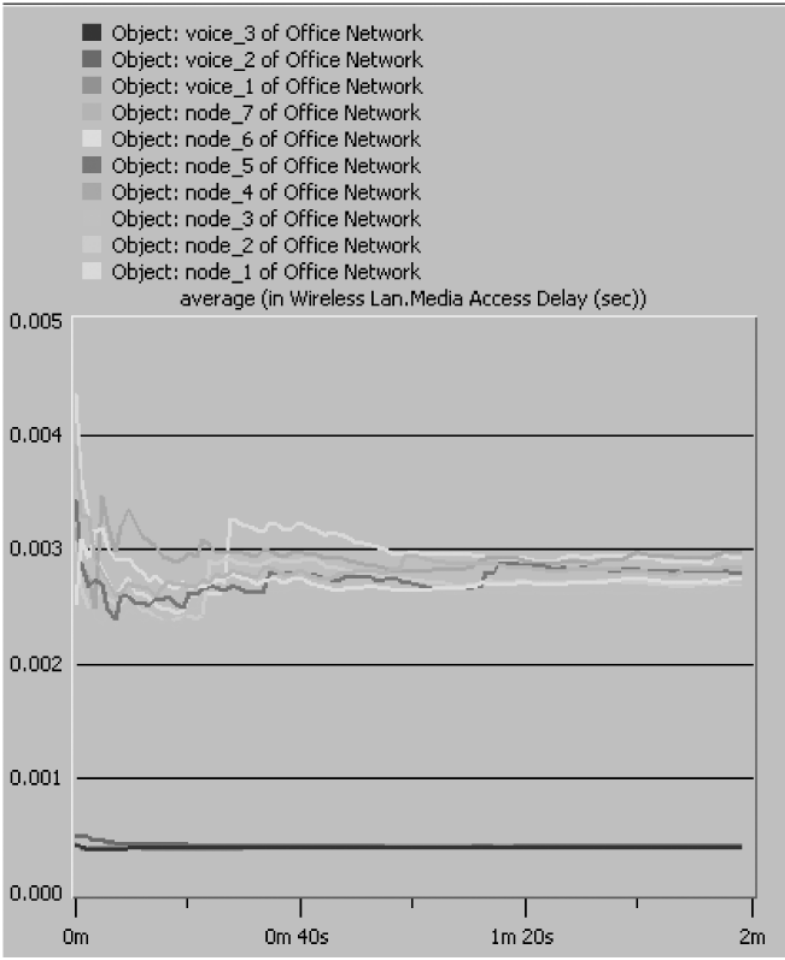

Figure 22. Media access delay with QoS, with roaming RTS.

In the other case, when roaming and RTS were applied, VoIP also showed better performance in the network with QoS. The statistics obtained are shown in Figures 21 and 22. The reason for the improvement in the second network is that, with QoS, voice traffic will not be delayed considerably, like the normal stations are when they need to send data. However, other types of traffic performance degraded, because the priority was reserved for VoIP handsets.

Table 3 summarizes the results collected form the five scenarios for the media access delay in the first case simulation. This was for neither case where either station has enabled roaming nor RTS frames.

\begin{tabular}{|c|c|c|c|c|c|}
\hline \multicolumn{2}{|r|}{ Scenario } & Voice & $\begin{array}{l}\text { Multi- } \\
\text { media }\end{array}$ & $\begin{array}{l}\text { Back- } \\
\text { ground }\end{array}$ & $\begin{array}{l}\text { Best } \\
\text { Effort }\end{array}$ \\
\hline \multicolumn{2}{|l|}{1} & 0.81 & 0.81 & 0.81 & 0.81 \\
\hline \multicolumn{2}{|c|}{2} & 0.150 & 0.168 & 0.257 & 0.344 \\
\hline \multicolumn{2}{|l|}{3} & 0.305 & 0.435 & 0.720 & 1.284 \\
\hline \multicolumn{2}{|c|}{4} & 0.083 & 0.103 & 0.141 & 0.212 \\
\hline \multirow{2}{*}{5} & (no QoS) & 1.772 & 1.772 & 1.772 & 1.772 \\
\hline & (with QoS) & 0.391 & 2.636 & 2.636 & 2.636 \\
\hline
\end{tabular}

Table 3. Media access delay in the first case (milliseconds). 
In scenario 1, all traffic types had the same media access delay value, since there was no QoS implemented.

As a result of programming QoS in the second layer, the voice traffic will have the lowest delay time compared to the other traffic types in scenarios from 2 to 5 . This implies that the voice traffic will not be delayed like the other traffic types before sending (i.e. it has a higher priority).

Table 4 shows the results collected from the same scenarios when applying roaming and allowing RTS/CTS frames. In general, the results between the two cases are comparable. However, one can notice that the voice packets have the highest priority when QoS is introduced to the wireless network.

\begin{tabular}{|l|c|c|c|c|}
\hline Scenario & Voice & $\begin{array}{c}\text { Multi- } \\
\text { media }\end{array}$ & $\begin{array}{c}\text { Back- } \\
\text { ground }\end{array}$ & $\begin{array}{c}\text { Best } \\
\text { Effort }\end{array}$ \\
\hline \hline 1 & 0.80 & 0.80 & 0.80 & 0.80 \\
\hline 2 & 0.111 & 0.140 & 0.211 & 0.307 \\
\hline 3 & 0.313 & 0.423 & 0.765 & 1.308 \\
\hline 4 & 0.083 & 0.107 & 0.160 & 0.201 \\
\hline 5 (no QoS) & 1.760 & 1.760 & 1.760 & 1.760 \\
\hline 5 (with QoS) & 0.565 & 3.794 & 3.794 & 3.794 \\
\hline
\end{tabular}

Table 4. Media access delay in the second case (millisecond).

\section{Conclusion}

The aim of the work presented in this paper is to program and simulate Enhanced Distributed Channel Access (EDCA) data priority method for the 802.11e standard. Although it is challenging to implement a voice communication with EDCA, it was adopted to be implemented over HCCA mainly because of HCCA's shortcomings. It is also because the EDCA is widely used and its operation is similar to the 802.11 DCF which is standard for media access. It has been shown that the introduction of QoS to the wireless network gives priority to highend profiles mainly voice and multimedia traffic types over the low-end profiles i.e. best effort and background traffic types. This work also concentrates on the effect of enabling stations roaming in WLAN and examines the effect of utilizing RTS/CTS management frames on the behavior of the network through examining the media access delay of the wireless stations and the load of the AP. It was found that roaming and RTS would help improving the WLAN performance in general.

\section{Acknowledgment}

This work is part of a Sultan Qaboos University Strategic Project \# ENG/ECED/04/01 and the authors would like to thank SQU for allocating funds for realizing this project.

\section{References}

[1] Federal Communication COMmission (FCC), Voice over Internet Protocol (VoIP): Consumer fact sheet. Federal Communication Commission (FCC)/National Association of Regulatory Utility Commissioners (NARUC) Task Force on VoIP Enhanced 911 Enforcement, April 2006.

(http://www.fcc.gov/cgb/consumerfacts/ voip.pdf).

[2] Colubris Network, Adding Voice Service to a Wi-Fi Network: Protecting QoS and Data Security. Colubris Network. Inc., May 9, 2004.

[3] Providing QoS in WLANs. How the IEEE 802.11e Standard QoS Enhancements Will Affect the Performance of WLANs. White Paper, Intel Corporation.

[4] WIKIPEDIA, Voice over IP. Article from Wikipedia, December 2006. (Available on:

http://en.wikipedia.org/wiki/VoIP).

[5] YU, JAMES, IEEE 802.11e QoS for Wireless LAN: A Research Direction. TDC Network Seminar, Multimedia Networking Research Laboratory, (2003).

[6] W. AL-MANDhery, Quality of Service for Voice over IP in Wireless Local Area Networks. pp. 47-48, M.Sc. Thesis, Electrical and Computer Engineering, Sultan Qaboos University, July 2006.

[7] S. Wietholter, C. Hoene, Design and Verification of an IEEE 802.11e EDCF Simulation Model in ns2.26. Report (TKN-03-19), Technical University of Berlin, Telecommunication Networks Group, 2003.

[8] Bejerano, Yigal and Bhatia, RandeEP, MiFi: A Framework for Fairness and QoS Assurance in Current IEEE 802.11 Networks with Multiple Access Points. The 23rd Conference of the IEEE Communications Society, (2004).

[9] Bermai, InC., FAQs: 802.11e and QoS. 390 Cambridge Avenue, 2004. 
[10] A. Al-NaAmany, H. Bourdoucen, A. Al-Sinani, W. AL-MENTHARI, Contribution to Improving Quality of Service in VoIP Wireless LAN. International Conference on Computer, Communication and Power, ICCCP'07, (February 2007), pp. 1921, SQU, Muscat.

Received: March, 2007 Accepted: December, 2007

Contact addresses:

A. Al-Naamany, H. Bourdoucen and W. Al-Menthari Department of Electrical and Computer Engineering Sultan Qaboos University P.O. Box 33

Al-Khodh, Muscat Oman, 123

e-mail: hadj@squ.edu.om

AHMED Al-NAAMANY is an Assistant Professor of electrical and computer engineering at Sultan Qaboos University. He received the Ph.D. from the University of Manchester Institute of Science and Technology, UK, 1995, the M.Sc. degree (EE-Computer Control) from Drexel University, United States, 1990 and B.Sc. degree (Multi Disciplinary Engineering) from Widener University, 1986, another B.Sc. (EE) from Widener University in 1986 (With Honors). He is a member of IEEE Computer and Control Societies. He has lectured computer architecture, computer control, computer networks and artificial antelligence applications. Dr. Al-Naamany is also recipient of Fulbright Scholarship from USIS, 1999.

HADJ BOURDOUCEN was born in El-Mahmel, Khenchela, Algeria. He received the B.S. degree in electrical engineering from the National Institute of Electricity and Electronics, INELEC, Algiers, in 1983 and the M.Sc. and Ph.D. degrees from Ecole Centrale de Lyon, France, in 1984 and 1987, respectively. From 1987 until 1990, he was teaching and conducting research at the University of Science and Technology Houari Boumedienne (USTHB), Algiers. He joined the Electronics and Communication Department of INELEC in 1990 for teaching undergraduate and graduate courses and conducting research on devices and circuits. In February 1998, he joined the Department of Electrical and Computer Engineering, Sultan Qaboos University, Muscat, Oman. His research activities and interests cover design, modelling and simulation of optical communication systems, computer networks and computerized instrumentation. Hadj Bourdoucen is an IEEE Senior member.

W. AL-MeNTHARI received his M.Sc. and B.Sc. degrees form Sultan Qaboos University, Sultanate of Oman, in 2001 and 2006 respectively. In October 2006, he joined the Ph.D. program in the University of Electro-Communications, Tokyo. He is currently in his second year of the Ph.D. program. His research interest includes the wireless local area networks, especially the quality of service in wireless Mesh networks and Ad-hoc wireless networks. 